\title{
FM-CW Snow RADAR
}

\author{
C.Abhishek ${ }^{1}$, N.Sasi Kiran ${ }^{2}$, M.Sudhakar Reddy ${ }^{3}$ \\ Dept., of Electronics and Communication Engineering SCSVMV University, Kanchipuram, India ${ }^{1,2,3}$
}

\begin{abstract}
Global warming is one of the most important issues facing humanity. The depth and extent of sea-ice are clear indicators of global warming. Snow cover on sea-ice is an important variable that affects the volume of sea-ice and indirectly global warming as a whole. This paper discusses the design of snow radar. Various models in the design of the snow radar are discussed. It concludes with the problems and the solutions to rectify the issues.
\end{abstract}

Keywords: FM-CW, RADAR, beat frequency, back ray scattering principle.

\section{INTRODUCTION}

The extent and thickness of sea-ice is an important indicator of global climate change, as it directly influences the energy transfer between the ocean and the atmosphere. An understanding of this integrated system, consisting of atmosphere, hydrosphere and cryosphere, can help us analyze global climate change. Snow cover on sea-ice is an important physical variable that impacts energy, physical and chemical processes that occur on the integrated system consisting of the Ocean, Sea-Ice and the Atmosphere. Shrinking of snow cover in the Polar Regions leads to a faster loss of sea-ice to melting. Sea-Ice melting poses a major threat to coastal ecosystems around the world by contributing to sea level increase. Physically, snow is a mixture of air, ice and liquid water.

Electromagnetically, snow is characterized by a dielectric constant that depends on the temperature, water content, density and shape of ice-particles and water inclusions. Dry snow has very little liquid water and mostly consists of ice and air. It is almost transparent to electromagnetic waves in the microwave region. Its dielectric constant lies between that of air $\left(\varepsilon_{\mathrm{r}}=1\right)$ and of ice $\left(\varepsilon_{\mathrm{r}}=3.15\right)$. Wet snow, on the other hand offers significant attenuation to electromagnetic waves and is also characterized by a much higher dielectric constant than dry snow. An appropriately designed radar sensor can be used to study snow cover over sea-ice; since the dielectric constant of air, snow and sea-ice are different. This dielectric contrast can be seen in the reflected signal. This helps in determining snow depth over sea-ice. An Ultra Wideband Frequency Modulated Continuous Wave Radar $r$ operates over a frequency range of 2 to $8 \mathrm{GHz}$. This bandwidth gives it a range resolution of about $4 \mathrm{~cm}$. In addition, operating in the lower microwave band allows the radar signal to propagate through snow without significant attenuation.

PRINCIPLE AND OPERATION OF FMCW RADAR Frequency Modulated Continuous Wave Radar transmits a frequency sweep, often called a chirp. The signal is reflected from distant targets and detected by the receiver where the return signal is mixed with a copy of the transmitted signal to determine the range of the target. The transmitted waveform has a time varying frequency $f(t)$ given by

$\mathrm{f}(\mathrm{t})=\mathrm{f}_{0}+\alpha . \mathrm{t}$

Where $f_{0}$ is the initial frequency, $\alpha$ is Rate of Change of Frequency and $\mathrm{T}$ is the Sweep time. The transmitted waveform travels to the target at distance $\mathrm{R}$ and returns after a time delay $\tau$ given by

$\mathrm{T}=2 \mathrm{R} / \mathrm{c}$ (Where $\mathrm{c}$ is the velocity of light in the medium)

Thus knowing the beat frequency and the radar parameters of $\mathrm{B}$ and $\mathrm{T}$, we can retrieve range information from the return signal. FMCW radars often store the beat frequency signal to allow for off-line processing using Fourier transform techniques. The Fast Fourier Transform (FFT) is the mathematical tool used to interpret the spectrum of the beat frequency signal in terms of radar range. The point target scenario can be extended to multiple targets also. For each target, there will be a return with a frequency corresponding to the distance. Each return will also have amplitude corresponding to the round trip attenuation to its specific target.

\section{METHODOLOGY}

The simulation parameters such as the sampling frequency and number of samples are initialized. Then the radar system information and target information are read in. The transmit waveform is then generated according to the source model. The simulation then follows two branches. In the first branch, the reflection coefficient is generated from the geophysical data. The reflected wave is then generated using the reflection coefficient. The system effects are then included by convolving this signal with the point spread function. This is then mixed with the transmit waveform and the reflected power is determined. Random noise is also incorporated at every range cell. In the second branch, the scattering coefficient is generated from the geophysical data.

Random phase noise is incorporated into the scattering coefficient and the backscattered wave is determined. Systems effects are included by convolving with the pointspread function. Multiple backscattered waves are generated and the mean backscattered power is computed as the mean power of the multiple backscattered waves mixed with the transmit wave. The total return power is computed as the sum of reflected and backscattered powers. The distance axis is computed after compensating for the variation in wave velocity due to difference in dielectric constant. This return power is then plotted versus the distance. This process can be represented by the flowchart below 


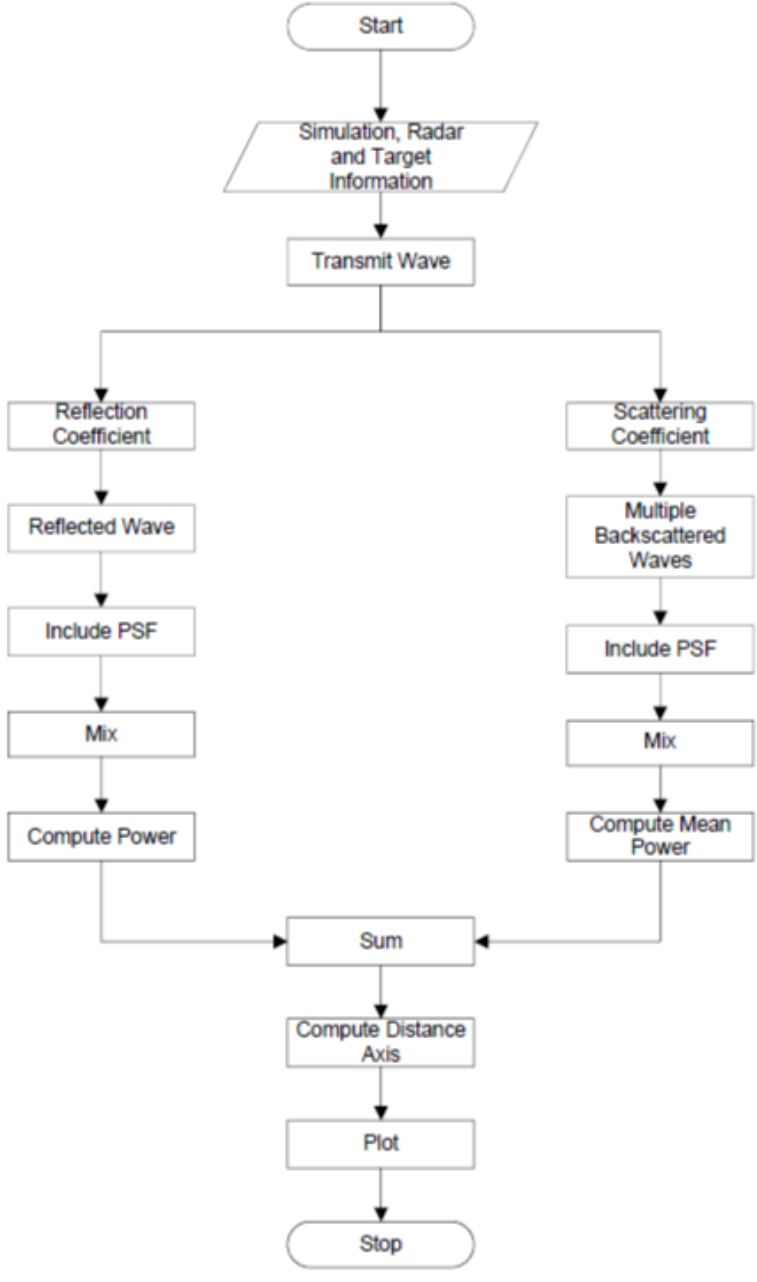

Fig.1. Flowchart

\section{FM-CW TRANSMISSION AND RECEPTION}

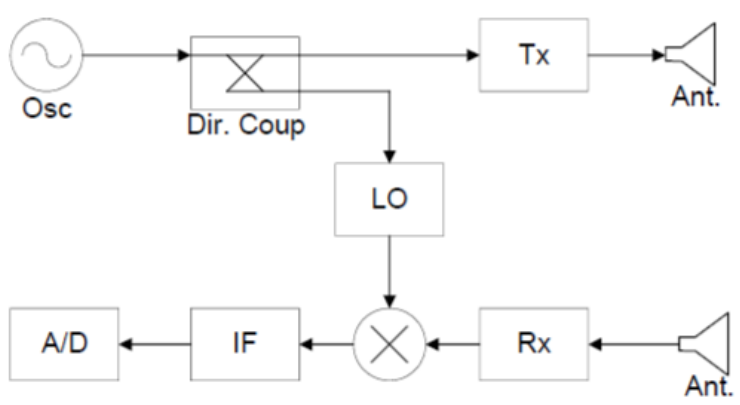

Fig.2. Radar System Functional Block Diagram

It mainly consists blocks namely Oscillator, Directional coupler, Transmitter, receiver, mixer, IF, low pass filter (LPF). The transmitter transmits the signal towards the target. Let the transmit signal frequency be $f_{t}$

\section{$\mathrm{f}_{\mathrm{t}}(\mathrm{t})=\mathrm{f}_{0}+\alpha \cdot \mathrm{t} \quad-(1)$}

This transmitted signal after hitting the target scatters back with some time delay $(\mathrm{t})$. Let the frequency of the received signal be $\mathrm{f}_{\mathrm{r}}$

$\mathrm{f}_{\mathrm{r}}(\mathrm{t})=\mathrm{f}_{0}+\alpha(\mathrm{T}-\mathrm{t})$

Mixing of these two signals produces sum and difference frequencies, $f_{t}+f_{r}$ and $f_{t}-f_{r}$. The resultant signal is then low pass filtered to remove the $f_{t}+f_{r}$ term.

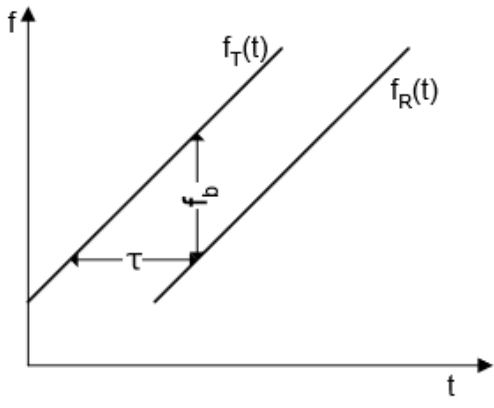

Fig.3. Beat Frequency

The term that remains is the beat frequency term $\mathrm{f}_{\mathrm{b}}$

$$
\begin{aligned}
\mathrm{f}_{\mathrm{b}} & =\mathrm{f}_{\mathrm{t}}-\mathrm{f}_{\mathrm{r}} \\
& =\alpha \cdot \mathrm{T} \\
& =2 \mathrm{RB} / \mathrm{Tc}
\end{aligned}
$$

Thus knowing the beat frequency and the radar parameters of $\mathrm{B}$ and $\mathrm{T}$, we can retrieve range information from the return signal. FMCW radars often store the beat frequency signal to allow for off-line processing using Fourier transform techniques. The Fast Fourier Transform (FFT) is the mathematical tool used to interpret the spectrum of the beat frequency signal in terms of radar range. The point target scenario can be extended to multiple targets also. For each target, there will be a return with a frequency corresponding to the distance. Each return will also have amplitude corresponding to the round trip attenuation to its specific target. These can be seen as peaks in the frequency domain. This can be further extended to spread targets. In this case, the return will not consist of only individual peaks but will have spread peaks corresponding to returns from other scatterers.

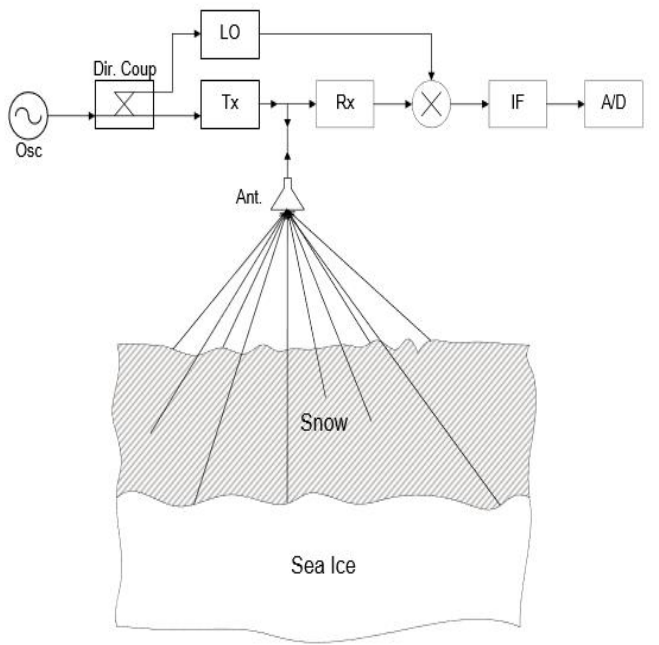

Fig.4. Model of Snow over Sea-Ice

The radar return signal from snow-covered sea-ice is characterized by a reflection term and a scattering term. The reflection term arises due to dielectric contrast between the layers that make up the medium. This reflected signal is modeled with the assumption that the layers are perfectly planar and that the antenna beam is a pencil beam. However, the surface is not perfectly smooth and the antenna has a finite bandwidth. This gives rise to the phenomenon of surface scattering. Surface scattering 
accounts for the fact that the antenna will pick up backscattered signals from angles other than nadir. Another phenomenon to be considered is volume scattering. Volume scattering occurs because the layers are not homogenous. Rather, snow consists of ice particles suspended in air. The surface of snow-covered sea-ice is not perfectly smooth but is characterized by roughness. This roughness is specified in terms of the rms height and the correlation length.

A signal ray incident normally on this rough surface does not undergo specular reflection. Instead, it is scattered at different angles. This would also be acceptable if we had a single ray incident on the target as it will only affect the amount of power returned to the receive antenna. However, as the transmit beam width is finite, rays scattered from the surface at angles other than nadir also return to the receive antenna. These might be mistaken for returns from nadir. The rms height is the standard deviation of the height of a surface. The correlation length is the distance at which the normalized autocorrelation of the surface height falls to (1/e) where e is the exponential constant (base of natural logarithms). As the rms height of a surface increases, it becomes rougher and as the correlation length of a surface increases it becomes smoother. Sometimes the ratio of the rms height to the correlation length called the surface slope is used as an indicator of roughness.

\section{SIMULATION PARAMETERS}

The primary simulation parameters are:

1. Sampling frequency / Sample time.

2. Number of samples / Chirp time.

3. Radar Parameters and Data.

4. Target Information.

1. Sampling Frequency: The sampling time has to be chosen to satisfy the Nyquist criterion, which requires that the sampling frequency be at least twice the maximum frequency of the sampled signal. As a general rule, simulations are usually run at a higher rate than the minimum required by Nyquist rate.

2. Number of Samples: The number of samples is a very important simulation parameter. The higher the number of samples the longer the simulation takes. In the case of the FMCW radar the number of samples is also directly related to the chirp time. The chirp time is the product of the number of samples and the sample time

Number of Samples $=$ Chirp Time $*$ Sampling Frequency 3. Radar Parameters and Data: Other inputs to the simulation include information about the radar system. These include radar parameters such as the start frequency and stop frequency of the radar, radar source models such as the amplitude and phase error models and radar system information such as the point spread function

4. Target Information: Information about the target such as delay line length and transfer function or the geophysical data are also inputs to the simulation. The geophysical data includes information about the constituent media of the target and its scattering parameters such as surface rms height and correlation length.

\section{Generation of Reflected Waveform and Power}

The reflected waveform is generated as the convolution of the transmit waveform with the effective reflection coefficient.

Transmit Chirp * Gamma = Reflected Wave

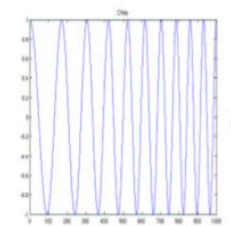

Transmit Chirp

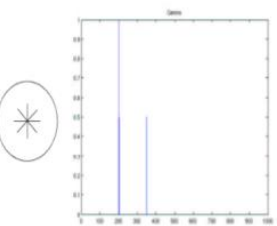

Gamma

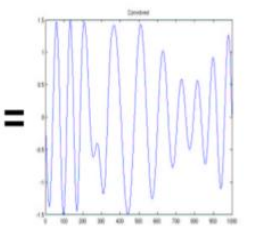

Reflected wave
The IF signal is then computed by mixing this reflected signal with the transmit waveform and convolving with the Point Spread Function. Then the Fast Fourier Transform of the IF signal is computed. This gives the reflected power vs. frequency. We know that range is directly proportional to frequency for FMCW radar as. Therefore, the obtained FFT is also a measure of reflected power as a function of distance. In order to incorporate random noise in to the simulation we now add random noise power at every range bin of the distance. The power level of this random noise is taken from the noise floor of actual radar data.

\section{GENERATION OF BACKSCATTERED WAVEFORM AND POWER}

The scattering coefficient is calculated as a function of scattering parameters. A random phase is added to this scattering coefficient. This is then convolved with the transmit wave to determine the backscattered wave. This process is repeated to generate multiple backscattered waves. Each of these waves is then 48 mixed with the transmit waveform and convolved with the point spread function. Then power is calculated. Finally, their mean is taken as the backscattered power versus range.

\section{CONCLUSION}

This paper discusses the design of snow radar. Various models in the design of the snow radar are discussed, various parameters and factors effecting the radar functioning are discussed in this paper. By rectifying all the design issues the efficiency of the radar can be increased.

\section{REFERENCES}

[1] Barber, D.G.; Fung, A.K.; Grenfell, T.C.; Nghiem, S.V.; Onstott, R.G.; Lytle, V.I.; Perovich, D.K.; Gow, A.J.; "The role of snow on microwave emission and scattering over first-year sea ice"; Geoscience and Remote Sensing, IEEE Transactions on, Volume: 36, Issue: 5 , Sept. 1998; Pages:1750 - 1763

[2] Tjuatja, S.; Fung, A.K.; Bredow, J.; "A scattering model for snowcovered sea ice"; Geoscience and Remote Sensing, IEEE Transactions on , Volume: 30 , Issue: 4, July 1992; Pages:804 - 810

[3] Yackel, J.J.; Barber, D.G.; "Validation of a snow water equivalence algorithm over landfast first-year sea ice using RADARSAT-1"; Geoscience and Remote Sensing Symposium, 2002. IGARSS '02. 2002 IEEE International, Volume: 1, 24-28 June 2002; Pages:234 236 vol. 1 
[4] Iacozza, J. and D.G. Barber. 1999.; "An examination of the distribution of snow on sea ice "; Atmosphere-Ocean, vol. 37(1); 21-51.

[5] Hallikainen, M.; Ulaby, F.; Abdelrazik, M.; "Dielectric properties of snow in the 3 to $37 \mathrm{GHz}$ range"; Antennas and Propagation, IEEE Transactions on, Volume: 34 , Issue: 11 , Nov 1986; Pages: 1329 - 1340

[6] Markus, T. and D. Cavalieri. ; "Snow depth distribution over sea ice in the Southern Ocean from satellite passive microwave data. Antarctic Sea Ice: Physical Processes, Interactions, and Variability." Antarctic Research Series 1998. 74:19-39. Washington, DC, USA: American Geophysical Union.

[7] Hallikainen, M.;"Review of the Microwave Dielectric and Extinction Properties of Sea Ice and Snow"; Geoscience and Remote Sensing Symposium, 1992. IGARSS '92. International, Volume: 2, 1992; Pages:961 - 965

[8] Gogineni S., Wong K., Krishnan S., Kanagaratnam P., Markus T. and Lytle V. "An Ultra-wideband Radar for Measurements of Snow Thickness over Sea Ice”; IGARSS'03; Toulouse, France, July 2003.

\section{BIOGRAPHIES}

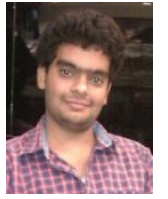

C.Abhishek is pursuing his under graduation in Scsvmv university Kanchipuram India .He has completed his research project in DRDO on inertial navigation system. He presented papers in international conferences. $\mathrm{He}$ completed his intern in DRDO. Currently he is doing his research on micro Doppler radar in NARL. He has undergone training in IIRS on remote sensing. His areas of interests are satellite communication, wireless systems, radar engineering. 